\title{
L'anemia nel paziente in dialisi peritoneale: indagine del Registro Toscano (RTDT)
}

\author{
Alberto Rosati ${ }^{1}$, Antonio Carlini ${ }^{1}$, Giuseppina Rosso ${ }^{1}$, Elisa Colombini ${ }^{1}$, Claudia del Corso ${ }^{2}$, Viviana Finato ${ }^{3}$ a nome dei \\ Referenti del Registro Toscano Dialisi e Trapianto \\ ${ }^{1}$ U.O. Nefrologia e Dialisi, USL2 Lucca, Lucca \\ ${ }^{2}$ U.O. Nefrologia e Dialisi, USL3 Pistoia, Pistoia \\ ${ }^{3}$ U.O. Nefrologia e Dialisi, USL11 Empoli, Empoli
}

\begin{abstract}
Anemia in peritoneal dialysis patients : a cohort study of Dialysis Tuscany Registry
Introduction: The optimization of anaemia treatment in dialysis patients is a main concern worldwide considering its health and financial implications. The use of erythropoiesis-stimulating agents (ESAs) usually allows good control of hemoglobin levels both in hemodialysis (HD) and in peritoneal dialysis (PD) patients. Although RCT are available only for HD and PD patients, strong evidences lack. Our aim was to evaluate anaemia management in
\end{abstract} HD and PD patients treated in Tuscany.

Methods: We analyzed data of 2440 patients registered by the Tuscany Registry of Dialysis and Transplantation (RTDT) and by the Tuscany Group of Peritoneal Dialysis in order to evaluate the differences in terms of anaemia correction, ESAs employment and iron status between HD and PD patients.

Results: PD patients showed higher and stable mean levels of $\mathrm{Hb}$ over the last 10 years whereas mean $\mathrm{Hb}$ levels in $\mathrm{HD}$ patient tended to lower in the last years according to guidelines suggestions. In PD patients resistance to erythropoietin therapy was lower and patients reached higher levels of $\mathrm{Hb}(11.6 \pm 1.3$ vs $11.3 \pm 2.4 \mathrm{p}=0.02)$ receiving a lesser weekly dose of ESAs ( 8000 vs $6000 \mathrm{UI} p=0.002)$. A lower percentage of patients received iron supplementation in PD (67.2 vs $33.1 \% p<0.01$ ) with significantly inferior levels of ferritin but similar levels of TSAT.

In PD patients the percentage of long acting ESAs was higher, the erythropoietin resistance index was lower and long acting ESAs are preferred. According to cost-effectiveness analyses the weekly cost of anaemia management is $14 \%$ lower in PD patients.

Conclusions: PD patients had a better correction with lower dosage of ESAs, lower iron supplementation and better cost-effectiveness ratio.

Keywords: Anaemia, ESAs, ERI, Peritoneal dialysis, Iron status, Cost-effectiveness

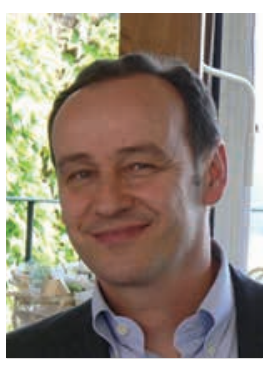

Alberto Rosati

\section{Introduzione}

Ĺanemia rappresenta una delle principali complicanze cliniche della malattia renale cronica (MRC), in tutti gli stadi del trattamento conservativo, durante il trattamento dialitico e nel post-trapianto di rene.

L'utilizzo di agenti stimolanti l'eritropoiesi (ESA) permette il trattamento

Accepted: January 19, 2015

Published online: May 28, 2015

Indirizzo per la corrispondenza:

Dr. Alberto Rosati

U.O. Nefrologia e Dialisi

Ospedale San Luca

55100 Lucca

a.rosati@usl2.toscana.it dell'anemia in tutti gli stadi della MRC con riduzione degli indici di morbidità e mortalità legati a questa complicanza, ma rappresenta uno dei principali "costi" economici di questa malattia $(1,2)$. Le Linee Guida di numerose società scientifiche nazionali e internazionali (3-5) hanno regolamentato e aggiornano continuamente le indicazioni alla prescrizione di questi farmaci, i target di emoglobina ed ematocrito da raggiungere e mantenere nel tempo, il rapporto costo/beneficio del trattamento stesso e gli eventuali effetti collaterali.

In letteratura, sono riportati pochi studi clinici sull'impiego degli ESA nei pazienti uremici cronici in trattamento di dialisi peritoneale (DP) e i maggiori studi clinici randomizzati sul rapporto fra ESA, anemia ed eventi cardiovascolari nei pazienti con MRC non hanno arruolato pazienti in $\operatorname{DP}(6,7)$. I protocolli di trattamento nel paziente in DP si basano soprattutto su indicazioni ricavate dai trial sui pazienti emodializzati ma, per molti versi, la gestione dell'anemia in DP è più simile a quella del paziente con MRC in terapia conservativa che a quella del paziente in emodialisi (HD) (8). II paziente in DP 
usa infatti dosi di ESA basse e ha, in genere, una funzione renale residua, una supplementazione di ferro modesta e una somministrazione di ESA che avviene generalmente per via sottocutanea.Questo studio nasce da un'analisi congiunta dei dati del Registro Toscano di Dialisi e Trapianto (RTDT) con i dati del Gruppo Toscano di Dialisi Peritoneale (GTDP). Lo studio, di natura osservazionale, ha avuto l'obiettivo di valutare la prevalenza dell'anemia in DP e in HD e predittori di trattamento con ESA, l'impatto relativo dell'anemia sulla sopravvivenza del paziente in DP e HD, l'assetto marziale in DP e HD, I'utilizzo degli ESA in DP, la resistenza agli ESA in DP vs HD e l'analisi di costo efficacia in DP vs HD.

\section{Materiali e metodi}

II RTDT, istituito dalla Regione Toscana, raccoglie dati relativi ai pazienti in trattamento sostitutivo residenti nella Regione Toscana fin dal 1996. Le informazioni di tipo clinico epidemiologico vengono raccolte nei singoli centri nefrologici sulla base di un'informativa e di un consenso esplicito da parte dei pazienti. La percentuale di adesione è molto elevata, mediamente $>96 \%$. II GTDP esegue indagini finalizzate su specifici topic clinici relativi al trattamento peritoneale.

L'analisi descrittiva si è basata su mediana e range interquartile (IQR) o su media e deviazione standard per le variabili normalmente distribuite e percentuali per le variabili categoriche. Chi-square test e Student's test o il Mann-Whitney test sono stati usati per confrontare rispettivamente proporzioni e medie. La regressione multinomiale logistica è stata utilizzata per valutare i predittori associati alla necessità di ESA, mentre l'analisi di regressione e l'analisi univariata della varianza sono state usate per definire le variabili che influenzano la sensibilità agli ESA.

\section{Risultati}

Dati demografici: alla fine del 2012, secondo i dati del RTDT (Tab. I), 2440 pazienti erano in trattamento dialitico nella Regione Toscana, di cui il $90 \%$ eseguiva HD e il $10 \%$ DP a domicilio; fra i pazienti in DP, la CAPD rappresentava il 40.3\% dei trattamenti, mentre l'APD il 59.7\%. La distribuzione dell'età era diversa in HD e in DP: nei pazienti in HD la \% di pazienti $>75$ anni era significativamente più alta che in $D P$, mentre le fasce d'età 46-65 e 66-75 erano più rappresentate in DP. La distribuzione per sesso non era invece diversa fra le due metodiche depurative con le femmine leggermente più rappresentate in DP.

Per quanto riguarda i livelli di emoglobinemia, i pazienti in DP presentavano valori mediamente superiori rispetto ai pazienti in HD (11.6 \pm 1.3 vs $11.3 \pm 2.4 p=0.02)$, inoltre il $30 \%$ dei pazienti in DP aveva livelli di $\mathrm{Hb}>12 \mathrm{~g} / \mathrm{dL}$ vs $26 \%$ in HD; anche l'andamento negli anni dei livelli medi è diverso fra le due metodiche; in HD si è osservata, negli ultimi anni, una
TABELLA I - Principali aspetti demografici, clinici e laboratoristici della popolazione in studio

\begin{tabular}{lcc}
\hline & HD & DP \\
\hline Età (anni) & $65.7 \pm 14$ & $64.7 \pm 14.0$ \\
Anzianità dialitica (mesi) & $70.4 \pm 76.8$ & $23.0 \pm 18.1$ \\
Ferritina (ng/mL) & $558(332-784)$ & $300(117-483)$ \\
Saturazione della & $33.0 \pm 14.4$ & $30.9 \pm 7.6$ \\
transferrina (\%) & & \\
Hb (g/dL) & $11.3 \pm 2.4$ & $11.6 \pm 1.3$ \\
Albuminemia (g/dL) & $3.6 \pm 0.5$ & $3.3 \pm 0.6$ \\
PCR (mg/L) & $10 \pm 14.5$ & $9.0 \pm 17.2$ \\
HDF on line & $27 \%$ & \\
APD & & $59.7 \%$ \\
Cardiopatia & $67.9 \%$ & $50.9 \%$ \\
Ipertensione & $35.0 \%$ & $92.8 \%$ \\
Vasculopatia cerebrale & $22.3 \%$ & $14.7 \%$ \\
Vasculopatia periferica & $37.1 \%$ & $33.5 \%$ \\
Diabete & $30.0 \%$ & $27.9 \%$ \\
Neoplasia & $29.0 \%$ & $10.3 \%$ \\
\hline
\end{tabular}

Media aritmetica \pm SD per variabili normalmente distribuite, mediana (IQR) per variabili non normalmente distribuite, percentuali per variabili categoriche.

tendenza alla riduzione dei valori medi di $\mathrm{Hb}$, cosa che non si è osservata in DP (Fig. 1).

\section{Impatto relativo dell'anemia sulla sopravvivenza in HD e DP}

Il rapporto fra anemia e mortalità nei pazienti in dialisi peritoneale non è stato sufficientemente indagato nei RCT. Studi osservazionali su casistiche anche molto ampie hanno evidenziato che il rischio di mortalità aumentava nella popolazione con $\mathrm{Hb}$ inferiore a 9-10 g/dL e che, per valori di emoglobina $>11-12 \mathrm{~g} / \mathrm{dL}$, non si verificava invece nessun incremento del rischio di ospedalizzazione e mortalità (9). Nella nostra casistica, l'analisi multivariata di Cox non dimostrava nessuna differenza statisticamente significativa della sopravvivenza a 3 anni, aggiustata per fattori di confondimento, nella popolazione suddivisa in base ai quartili di $\mathrm{Hb}$, anche se $\mathrm{i}$ pazienti appartenenti al primo quartile $(\mathrm{Hb}<10.6)$ avevano una sopravvivenza a 3 anni < di 8 punti rispetto ai pazienti appartenenti al quarto quartile ( $\mathrm{Hb}>12.3)$.

Anche trasportare sulla dialisi peritoneale i concetti relativi alla dose di ESA necessaria per raggiungere certi livelli di $\mathrm{Hb}$ e alla relazione che questa ha con il rischio di mortalità 


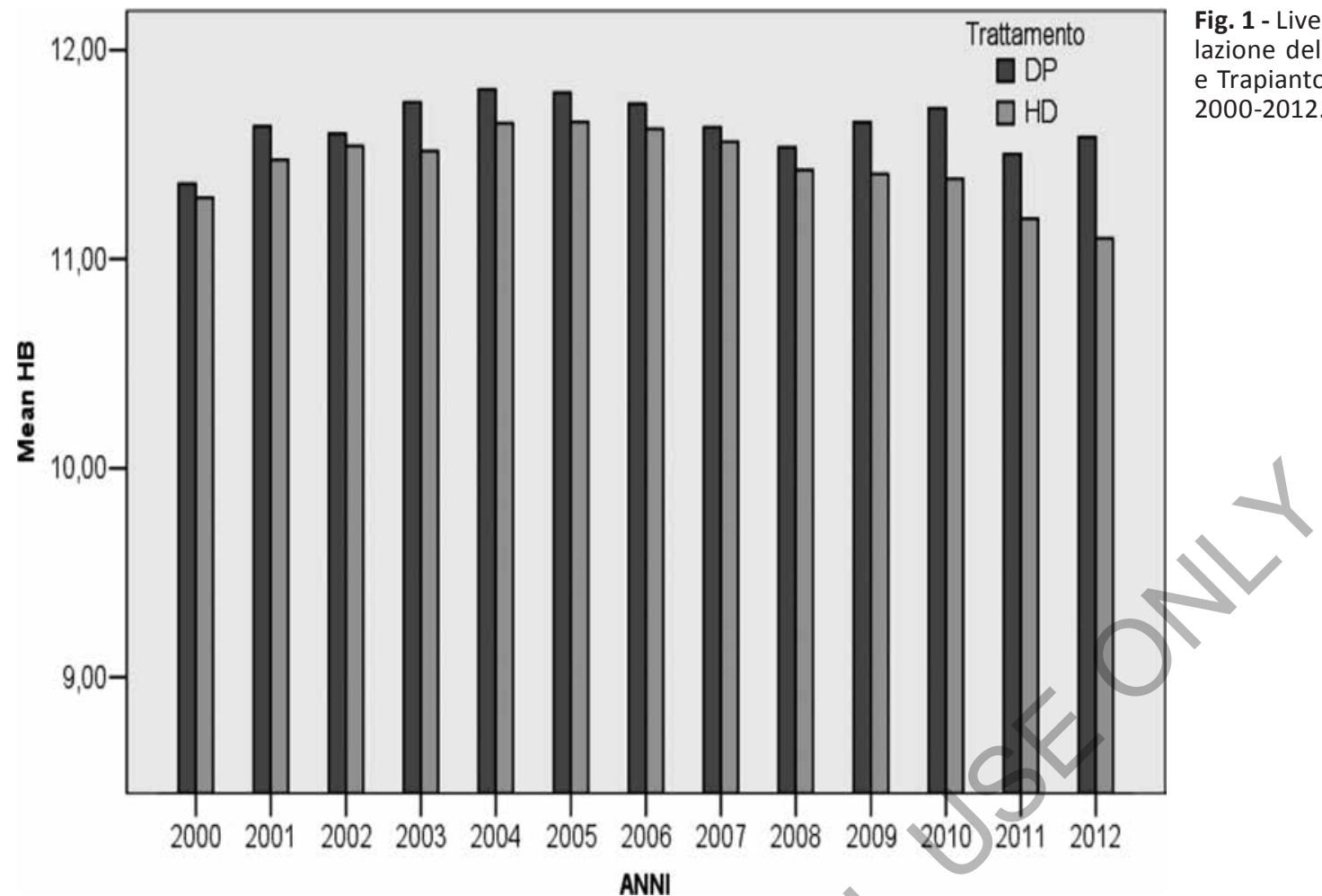

non è affatto semplice. Per i pazienti in HD è dimostrabile una relazione lineare fra dosaggi di ESA e rischio di mortalità; tale relazione, nel paziente in DP, è dimostrabile solo per dosaggi $>15000 \mathrm{UI} / \mathrm{sett}$. (1), dosaggi che, nella pratica clinica, molto raramente si realizzano in un paziente in DP. In uno studio comparativo fra HD e DP si dimostrava un aumento del rischio di mortalità nei pazienti in HD e DP che ricevevano una dose settimanale di ESA >15000 UI/sett. e chela dose di ESA si correlava con il rischio di morte nella popolazione in HD ma non in quella in DP (10).

\section{Assetto marziale in DP}

Per quanto riguarda l'uso della terapia marziale, il $67.2 \%$ dei pazienti in HD riceveva una supplementazione di ferro rispetto a un $33.1 \%$ dei pazienti in DP $(p<0.01)$; inoltre, nei pazienti in HD, questa era praticata esclusivamente per via parenterale, mentre, in DP, per via orale. Se si valutano i livelli di ferritinemia in HD e DP, questi risultavano significativamente superiori in HD rispetto alla DP, anche dopo aggiustamento per fattori di confondimento $524 \pm 450$ vs $369 \pm$ $312 \mathrm{ng} / \mathrm{mL}(\mathrm{p}<0.001)$, mentre i livelli medi di saturazione della transferrina (TSAT) erano quasi sovrapponibili fra le due metodiche dialitiche, anche se rapportati ai quartili di emoglobina. Se restringiamo il campo ai soli pazienti in trattamento con ESA, la \% di pazienti con ferritinemia $>500$ $\mathrm{ng} / \mathrm{mL}$ era molto più elevata in $\mathrm{HD}$ rispetto alla DP (43\% vs $10.5 \% \mathrm{p}<0.01)$, mentre, al contrario, solo un $11 \%$ dei pazienti in HD aveva valori $<100 \mathrm{ng} / \mathrm{mL}$ contro un $30 \%$ dei pazienti in DP. Relativamente ai livelli di TSAT, il 73\% dei pazienti in DP e il 59\% di quelli in HD aveva livelli compresi fra il $30 \%$ e il $50 \%$. La popolazione in DP con ferritinemia $<100 \mathrm{ng} / \mathrm{mL}$ apparirebbe come una popolazione che, secondo le Linee Guida, ha una forte indicazione alla supplementazione di ferro; in realtà, solo il $42 \%$ di questi pazienti riceveva supplementi di ferro, pur mantenendo in gran parte una TSAT costantemente adeguata.

\section{Utilizzo degli ESA in DP}

Analizzando la percentuale di pazienti non trattati con ESA in DP e HD, nei periodi 2000-2010 e 2011-2013, questa era significativamente più bassa nei pazienti in DP che in quelli in HD in entrambi i periodi in tutte le fasce d'età esaminate.

I predittori indipendenti di terapia con ESA in DP (dopo avere aggiustato i dati per BSA, PET, albumina, PCR, ecc.) risultavano essenzialmente il diabete mellito, in analogia con quanto riportato da altri studi (15), con un aumento del rischio di 6 volte $(p=0.005, H R=6.51 \mathrm{Cl}=1.78-23.78)$, e la vasculopatia periferica, con un aumento del rischio di tre volte ( $p=0.049, \mathrm{HR}=3.004 \mathrm{Cl}=1.005-8.98)$. Sia il GFR residuo che la diuresi risultavano molto vicini alla significatività (Tab. II).

Confrontando la dose settimanale media di ESA nelle due metodiche dialitiche, si dimostrava una dose media di ESA significativamente maggiore in HD rispetto alla DP (8000 vs $6000 \mathrm{UI} / \mathrm{sett} . \mathrm{p}=0.002$ ) e questo in linea con casistiche eu- 
TABELLA II - Predittori indipendenti di terapia con esa in DP

\begin{tabular}{lcccc}
\hline & $\mathbf{p}$ & HR & \multicolumn{2}{c}{$\begin{array}{c}\text { Intervallo di } \\
\text { confidenza al 95\% }\end{array}$} \\
\hline Diuresi & 0.067 & 0.923 & 0.895 & 1.002 \\
GFR & 0.054 & 0.826 & 0.681 & 1.003 \\
$\begin{array}{l}\text { Vasculopatia } \\
\text { periferica }\end{array}$ & $\mathbf{0 . 0 4 9}$ & $\mathbf{3 . 0 0 4}$ & $\mathbf{1 . 0 0 5}$ & $\mathbf{8 . 9 8 2}$ \\
$\begin{array}{l}\text { Diabete } \\
\text { Sedentarietà }\end{array}$ & $\mathbf{0 . 0 0 5}$ & $\mathbf{6 . 5 0 7}$ & $\mathbf{1 . 7 8 1}$ & $\mathbf{2 3 . 7 8 0}$ \\
& 0.060 & 2.611 & 0.959 & 7.114 \\
\hline
\end{tabular}

Regressione Multinomiale Logistica, aggiustato per BSA, PET, Albumina, PCR, Beta2 microglobulina, Ferritina, TSAT, Sesso, Cardiopatia, Ipertensione, Vasculopatia cerebrale, BPCO, Epatopatia cronica, Neoplasia maligna, Fumo, Obesità, Sedentarietà, Assunzione di ACEI/ARB, Betabloccanti, Calcitriolo, Paracalcitolo, Terapia marziale, Tecnica dialitica.

TABELLA III - Predittori associati con il livello di sensibilità agli ESA in HD e DP

\begin{tabular}{lcc}
\hline & HD & DP \\
\hline Saturazione transferrina & $p$ & $p$ \\
Ferritina & 0.017 & 0.199 \\
PCR & 0.343 & 0.026 \\
Albuminemia & 0.001 & 0.048 \\
GFR & 0.0001 & 0.925 \\
\hline
\end{tabular}

Regressione Multinomiale Logistica, aggiustato per Età, Sesso, Anzianità dialitica, Cardiopatia, Ipertensione, Diabete, Vasculopatia periferica, Neoplasia maligna, Terapia marziale, Tecnica dialitica.

ropee (11). Studi statunitensi riportano differenze molto più eclatanti, ma partendo da dosi settimanali di ESA molto più elevate (12). Un diverso fabbisogno di ESA generalmente esprime una diversa risposta al trattamento. Si è soliti valutare la risposta con il cosiddetto ERI (erythropoietin resistance index), che altro non è che la dose settimanale di ESA aggiustata per il peso e per i livelli di $\mathrm{Hb}$ raggiunti (13). I pazienti appartenenti al quartile più alto di ERI sono quelli che rispondono meno al trattamento. Nella nostra casistica, i livelli di ERI erano mediamente $>40 \%$ nei pazienti in $\mathrm{HD}$, mentre il fabbisogno di ESA era $>30 \%$. Questa differenza è da attribuire ai più elevati livelli di $\mathrm{Hb}$ che mediamente si raggiungono nei pazienti in terapia con ESA in DP rispetto all'HD. Se esaminiamo i predittori di risposta agli ESA in HD e DP, osserviamo delle differenze sostanziali: in HD la TSAT era un ottimo predittore di risposta, mentre non lo era la ferritina; in DP la ferritina prediceva molto meglio la risposta rispetto alla TSAT (Tab. III), la PCR era un predittore indipendente di risposta agli ESA sia in DP che in HD, mentre l'albuminemia giocava un ruolo determinante solo nei pazienti in HD; infine, la funzione renale residua si correlava significativamente con la risposta agli ESA nei pazienti in DP. Suddividendo la popolazione dei pazienti in DP per i quartili di ERI i pazienti appartenenti al quarto quartile avevano una sopravvivenza significativamente inferiore, anche se la differenza era ai limiti della significatività $(p<0.05)$.

\section{ESA long acting vs short acting}

Negli ultimi anni si è assistito all'introduzione dei biosimilari di alcuni ESA per il trattamento dell'anemia del paziente in dialisi. In Toscana in particolare le politiche sanitarie sono state orientate a una massiccia diffusione dell'utilizzo di queste molecole. La delibera più recente, emanata nel Marzo del 2013, poneva obiettivi importantialle Aziende Sanitarie sull'impiego dei biosimilari: I'ESA biosimilare doveva rappresentare almeno il $65 \%$ del totale dell'epoietina alfa e il $15 \%$ dell'epoietina beta. I nostri dati hanno confermato che la penetrazione delle molecole biosimilari in HD è stata massiccia con \% mediamente maggiori rispetto a quelle indicate dalle delibere, laddove gli ESA a breve emivita (epoietina alfa ed epoietina beta) rappresentavano oltre il $70 \%$ del consumo totale di eritropoietina.

Per quanto riguarda la dialisi peritoneale, le molecole a lunga emivita (Darbepoetina ed Epoietina beta pegilata) rappresentavano oltre la metà del totale, con la Darbepoetina che era oltre il $40 \%$, con una percentuale di biosimilari relativamente bassa.

Se, dopo aver aggiustato per le variabili di confondimento, si esaminavano i livelli di ERI, in HD e in DP, per le molecole long acting vs molecole short acting, per le prime si registrava un ERI $<38 \%$ in DP rispetto all'HD ( $p<0.001)$. Anche per le molecole short acting, i livelli di ERI erano decisamente inferiori in DP $(-31 \%, p<0.01)$; per le molecole short acting dobbiamo, però, considerare che buona parte del vantaggio in termini di ESA resistenza è dovuto al passaggio dalla via di somministrazione endovenosa alla via di somministrazione sottocutanea. È, infatti, noto che, per una molecola short acting, il passaggio alla somministrazione sottocutanea determina un risparmio del $20-30 \%$ della dose $(14,15)$. Per le molecole long acting non sono invece riportate differenze significative di dosaggio per le due vie di somministrazione $(16,17)$.

\section{Farmacoeconomia}

Se si confrontano i dati di gestione dell'anemia nei pazienti in CKD terminale in trattamento dialitico (HD/DP) in Toscana si evidenziano (Tab. IV):

- 2201 pazienti in HD, di cui il $91 \%$ trattato con ESA e il $68 \%$ con terapia marziale endovenosa;

- 244 pazienti in DP, di cui il $78 \%$ trattato con ESA e il $31 \%$ con terapia marziale;

- $\quad$ il tipo di ESA era long acting nel $23 \%$ dei pazienti in HD rispetto al $58 \%$ in DP; 
TABELLA IV - Analisi farmacoeconomica del costo del trattamento dell'anemia in HD e DP

\begin{tabular}{lcc}
\hline & HD & DP \\
\hline Consistenza & $2201 \mathrm{pz}(90 \%)$ & $244 \mathrm{pz}(10 \%)$ \\
ESA trattati & $2009(91 \%)$ & 191 (78.3\%) \\
Terapia Marziale & $68 \%(\mathrm{ev})$ & $31 \%$ (os/ev) \\
Terapia Marziale & $1 \mathrm{f} / \mathrm{sett}$. & $1 \mathrm{f} / \mathrm{sett} .-1 \mathrm{c} / \mathrm{die}$ \\
dose media & & \\
Tipi di ESA \% & Long acting 23\% & Long acting 58\% \\
& Short acting orig. 45\% & Short acting orig. 31\% \\
& Short acting bios. 32\% & Short acting bios. 11\% \\
Dose media & $8000 \mathrm{U}$ & $6000 \mathrm{U}$ \\
settimanale & & \\
ERI (indice di & & 8.7 \\
resistenza) & 11.6 & 9.1 \\
Short acting & 12.8 & 0 \\
Long acting & 5 min/inf/som & 21.20 euro/pz \\
Costi prep./ & & \\
Sommin & 24.75 euro/pz & \\
Costo/settimana & & \\
\hline
\end{tabular}

Riferimento Costi: Gara ESTAV Distribuzione Diretta.

- la dose media settimanale era di $8000 \mathrm{UI}$ in HD rispetto a $6000 \mathrm{UI}$ in DP;

- l'ERI era più basso in DP che nell'HD sia per gli ESA long acting che per quelli short acting;

- il costo settimanale per paziente risultava inferiore del $14.4 \%$ nei pazienti in DP rispetto ai pazienti in HD.

\section{Discussione}

I dati sui quali si basano le attuali indicazioni sulla gestione dell'anemia nei pazienti in DP sono o puramente osservazionali oppure traslati da trial eseguiti su pazienti in trattamento emodialitico. Gli studi randomizzati eseguiti su pazienti in DP sono infatti caratterizzati da una scarsa numerosità e non forniscono, quindi, evidenze significative. Se noi osserviamo le caratteristiche dell'anemia del paziente in DP ci possiamo rendere conto di come, in realtà, questa abbia molte più affinità con l'anemia del paziente con CKD che non con quella del paziente in HD. In effetti, le dosi di ESA sono basse, la via di somministrazione è quasi sempre sottocutanea, vi è, in genere, una funzione renale residua e la supplementazione di ferro è modesta e soprattutto per via orale. Caratteristiche che, come possiamo vedere, ci avvicinano molto al paziente con CKD. Trasferire suggerimenti e protocolli utilizzati in HD è, quindi, del tutto arbitrario.

Come evidenziato da altri studi osservazionali (12) mediamente in DP si raggiungono livelli di $\mathrm{Hb}$ più elevati che in
HD e il fenomeno di progressiva riduzione dei livelli medi di $\mathrm{Hb}$ osservato negli ultimi anni nei pazienti in HD come conseguenza del modificarsi delle indicazioni delle Linee Guida è stato molto più sfumato in DP. Anche il rapporto fra livelli di $\mathrm{Hb}$ e mortalità così enfatizzato nei pazienti in HD non ha trovato nessuna dimostrazione convincente in DP. In assenza di trial randomizzati, i più importanti studi osservazionali hanno confermato solo un incremento di rischio per valori di $\mathrm{Hb}<9$ $10 \mathrm{~g} / \mathrm{dL}$ ma nessun rischio aggiuntivo quando l'Hb sale oltre i $12.5-13 \mathrm{~g} / \mathrm{dL}$ rispetto al riferimento (11-12 g/dL). Nella nostra casistica i pazienti appartenenti al $1^{\circ}$ quartile di $\mathrm{Hb}$ avevano una sopravvivenza a 3 anni inferiore di 8 punti, ma il dato non raggiungeva la significatività statistica.

Altro dato di interesse era la dose di ESA utilizzata per raggiungere questi livelli $\mathrm{di} \hat{\mathrm{Hb}}$. In linea con quanto riportato nella letteratura internazionale, i pazienti in DP utilizzavano dosi di ESA mediamente inferiori del $25 \%$; se si considera che i livelli di $\mathrm{Hb}$ raggiunti erano mediamente più elevati, ne deriva che i livelli di ERI erano mediamente superiori del $40 \%$ in HD.

Non vi è alcun dúbbio che l'assetto marziale e la gestione della supplementazione di ferro giocano un ruolo determinante nella gestione dell'anemia. Di nuovo le caratteristiche dell'assetto marziale in DP e in HD erano profondamente diverse. La percentuale di pazienti che ricevevano una supplementazione di ferro era meno della metà in DP, dove era praticata quasi esclusivamente per via orale. La ferritinemia era più bassa, mentre la TSAT era sostanzialmente sovrapponibile. Fra i pazienti in trattamento con ESA, ben il $30 \%$ aveva valori di ferritinemia $<100$ nonostante una supplementazione di ferro per via orale. Ne possiamo dedurre che, nonostante la possibile indicazione alla terapia marziale endovenosa, questa veniva praticata in una \% molto bassa di pazienti certamente per i problemi organizzativi che ciò comporta. Possiamo speculare che un più esteso uso della supplementazione di ferro per via endovenosa avrebbe potuto ridurre ulteriormente l'indicazione e il dosaggio della terapia con ESA. La ferritina era, infatti, un predittore di risposta agli ESA in DP mentre non lo era la TSAT, al contrario di quanto si osservava in HD, il che sottolinea il migliore uso del ferro in DP. È probabile che i livelli di Epcidina giochino un ruolo fondamentale nello spiegare tali differenze $(18,19)$.

Diabete e vasculopatia periferica rappresentavano i fattori di rischio principali associati alla necessità di terapia con ESA (15); molto vicini alla significatività statistica erano anche la diuresi e il GFR residuo (Tab. II). I principali fattori che influenzavano la resistenza al trattamento e che, quindi, predicevano il fabbisogno di ESA erano, invece, la Ferritinemia e il GFR residuo (Tab. III).

Le molecole long acting, in particolare la Darbepoetina, erano quelle più utilizzate per il trattamento. Nel confronto DP vs HD il vantaggio a favore della DP in termini di responsività al trattamento con ESA era più evidente per le molecole a lunga emivita che non per quelle a breve emivita. Questa è una delle componenti che, in una semplice analisi farma- 
coeconomica, ci permetteva di affermare che il costo della gestione dell'anemia nel paziente in DP era del $14 \%$ inferiore rispetto a quella del paziente in HD.

In conclusione, i suggerimenti delle Linee Guida per la gestione dell'anemia nel paziente in DP, in gran parte mutuati dall'HD, sono poco supportati da evidenze scientifiche. Il paziente in DP presenta un assetto marziale e una sensibilità agli ESA che lo avvicinano molto di più al paziente in terapia conservativa. La funzione renale residua, la continuità del trattamento e la migliore biocompatibilità del sistema sono probabilmente le ragioni alla base di questa differenza. Ne deriva un rapporto costo efficacia del trattamento dell'anemia in DP molto più vantaggioso che in HD. Questo è uno dei motivi che contribuisce a rendere la dialisi peritoneale economicamente più vantaggiosa rispetto all'emodialisi e di cui bisogna tenere conto quando si fanno delle valutazioni di farmacoeconomia.

\section{Disclosures}

Financial support: No financial support was received for this submission.

Conflict of interest: The authors have no conflict of interest.

\section{Bibliografia}

1. Molnar MZ, Mehrotra R, Dong U, Kovesdy CP, Kalantar-Zadeh K. Association of Hemoglobin and Survival in Peritoneal Dialysis Patients. Clin J Am Soc Nephrol. 2011;6:1973-81.

2. Regidor DL, Kopple JD, Kovesdy CP, et al. Associations between changes in hemoglobin and administered erythropoiesisstimulating agent and survival in hemodialysis patients. J Am Soc Nephrol. 2006;17:1181-91.

3. KDIGO Clinical Practice Guideline for Anemia in Chronic Kidney Disease. Kidney Int Suppl. 2012;2:279-335.

4. Locatelli F, Bárány $\mathrm{P}$, Covic A, et al. Kidney Disease: Improving Global Outcomes guidelines on anaemia management in chronic kidney disease: a European Renal Best Practice position statement. Nephrol Dial Transplant. 2013;28:1346-59.

5. KDOQI Clinical Practice Guidelines and Clinical Practice Recommendations for Anemia in Chronic Kidney Disease. Am J Kidney Dis. 2006;47:S11-145.

6. Singh AK, Szczech L, Tang KL, et al. Correction of anemia with epoetin alfa in chronic kidney disease. N Engl J Med. 2006;355:2085-98.

7. Pfeffer MA, Burdmann EA, Chen CY, et al. A trial of darbepoetin alfa in type 2 diabetes and chronic kidney disease. N Engl J Med. 2009;361:2019-32.

8. Palmer SC, Navaneethan SD, Craig JC, et al. Meta-analysis: Erythropoiesis-stimulating agents in patients with chronic kidney disease. Ann Intern Med. 2010;153:23-33.

9. Shuling L, Foley RN, Collins AJ. Anemia, hospitalization, and mortality in patients receiving peritoneal dialysis in the United States. Kidney Int. 2004;65:1864-9.

10. Duong $U$, Kalantar-Zadeh K, Molnar MZ, et al. Mortality Associated with Dose Response of Erythropoiesis-Stimulating Agents in Hemodialysis versus Peritoneal Dialysis Patients. Am J Nephrol. 2012;35:198-208.

11. Raoa A, Gilga J, Williams A. UK Renal Registry $15^{\text {th }}$ Annual Report: Chapter 6 Haemoglobin, Ferritin and Erythropoietin Amongst UK Adult Dialysis Patients in 2011: National and Centre-Specific Analyses. Nephron Clin Pract. 2013;123 (Suppl. 1):125-50.

12. Snyder JJ, Foley RN, Gilbertson DT, Vonesh EF, Collins AJ. Hemoglobin levels and erythropoietin doses in hemodialysis and peritoneal dialysis patients in the United States. J Am Soc Nephrol. 2004;15:174-9.

13. Kalantar-Zadeh K, Lee GH, Miller JE, et al. Predictors of hyporesponsiveness to erythropoiesis-stimulating agents in hemodialysis patients. Am J Kidney Dis. 2009;53:823-34.

14. Lo WK. Latest strategy in renal anemia management in peritoneal dialysis patients. Perit Dial Int. 2008;28(Suppl. 3): S76-80.

15. Dúng U, Mehrotra R, Molnar MZ, et al. Glycemic control and survival in peritoneal dialysis patients with diabetes mellitus. Clin J Am Soc Nephrol. 2011;6:1041-8.

16. Feriani M, De Meester JM, McMahon LP, et al. Extended dosing of darbepoetin alfa in peritoneal dialysis patients. BMC Nephrol. 2011;12:13.

17. Selby NM, Fonseca SA, Fluck RJ, Taal MW. Hemoglobin variability with epoetin beta and continuous erythropoietin receptor activator in patients on peritoneal dialysis. Perit Dial Int. 2012;32(2):177-82.

18. Eguchi A, Mochizuki T, Tsukada M, et al. Serum Hepcidin Levels and Reticulocyte Hemoglobin Concentrations as Indicators of the Iron Status of Peritoneal Dialysis Patients. Int J Nephrol. 2012;2012:239476.

19. Zaritsky J, Young B, Wang HJ, et al. Hepcidin-a potential novel biomarker for iron status in chronic kidney disease. Clin J Am Soc Nephrol. 2009;4(6):1051-6. 